\title{
G-CSF and GM-CSF Are Different. Which One Is Better for COVID-19?
}

\author{
Hillard M. Lazarus ${ }^{a}$ Robert Peter Gale ${ }^{b}$ \\ a Department of Medicine, Division of Hematology and Oncology, Case Western Reserve University, \\ Cleveland, $\mathrm{OH}, \mathrm{USA} ;{ }^{\mathrm{b}}$ Centre for Haematology Research, Department of Immunology and Inflammation, \\ Imperial College London, London, UK
}

Development of molecularly cloned myeloid hematopoietic growth factors (e.g., granulocyte colony-stimulating factor [G-CSF] and granulocyte-macrophage colonystimulating factor [GM-CSF]) more than 30 years ago increased safety and efficacy of intensive chemotherapy and radiation therapy by reversing damage to bone marrow function, thereby decreasing infections and bleeding and shortening hospitalizations. These drugs were also used to mobilize bone marrow hematopoietic progenitor cells into the blood facilitating their use as a graft for hematopoietic cell transplants. Other proposed uses included increasing efficacy of anti-leukemia chemotherapy and treating persons exposed to high doses of acute wholebody ionizing radiation $[1,2]$. Safety and efficacy of Gand GM-CSFs, typically given intravenously or subcutaneously, are well-known. Practice guidelines and consensus statements on their use are available from many medical societies and organizations including the American Society of Clinical Oncology (ASCO), the European Society of Medical Oncology (ESMO), the American Society of Hematology (ASH), and the National Comprehensive Cancer Network (NCCN).

G-CSF (e.g., filgrastim and pegfilgrastim, and their biosimilars) and GM-CSF (e.g., sargramostim) are the two most common types of hematopoietic growth factors. These drugs (proteins) are sometimes thought as being interchangeable. This is wrong. Structure, receptors, receptor distribution, and biologic effects of these pro-

karger@karger.com
www.karger.com/aha
Karger ${ }^{\prime /}$

teins differ substantially. Filgrastim is a $19-\mathrm{kDa}$ protein produced in E. coli which is not glycosylated. In contrast, sargramostim, a mixture of three GM-CSFs with molecular weights of $19.5,16.8$, and $15.5 \mathrm{kDa}$, is a glycosylated protein produced in S. cerevisiae. Glycosylation adds stability and degradation resistance [3]. Receptors for filgrastim and sargramostim belong to the cytokine receptor super-family. The G-CSF receptor (G-CSFR; CD114) is a homo-oligo-dimer, whereas the GM-CSF receptor (GM-CSFR; CD116) is a hetero-oligo-dimer sharing a $\beta$-chain with the IL-3 and IL- 5 receptors. The G-CSFR is expressed primarily on neutrophils and bone marrow precursor cells. The GM-CSFR, more widely expressed than the G-CSFR, is present on neutrophils, monocytes, eosinophils, dendritic cells, basophils, and, possibly, Bcells, whereas the G-CSFR is expressed only on neutrophils and monocytes [4]. Differences in receptor expression account for most of the biologic differences between filgrastim and sargramostim. Importantly, G-CSF is the dominant colony-stimulating factor (CSF) released from lung cells in response to pro-inflammatory cytokines [5].

G-CSF is the most widely used molecularly cloned hematopoietic growth factor (shown in Fig. 1). GM-CSF has a broader range of biologic activities than G-CSF as well

Dedicated to Academician Andrei Vorobiev of the Russian Federation, the 2 nd human to receive GM-CSF, who died recently.
Hillard M. Lazarus

Hillard.Lazarus@case.edu 


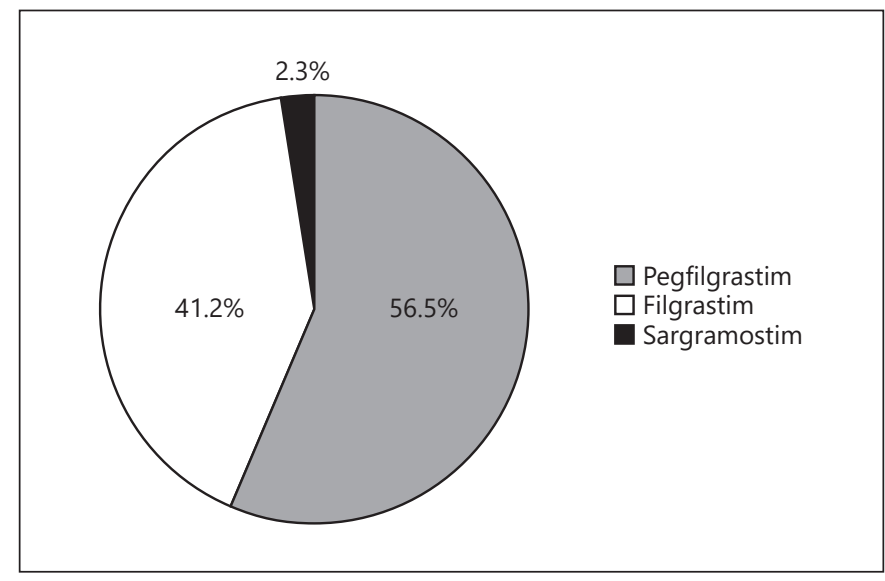

Fig. 1.2019 US market share distribution of hematopoietic growth factors. Data include pegfilgrastim (Neulasta ${ }^{\circledR}$, FulphilaTM, Udenyca ${ }^{\circledR}$ ), filgrastim (Neupogen ${ }^{\circledR}$, Zarxio $^{\circledR}$, Granix $^{\circledR}$, Nivestym ${ }^{\circledR}$ ), and sargramostim (Leukine ${ }^{\circledR}$ ). Source: IQVIA National Sales Perspectives $^{\circledR}$, June $5,2020$.

as anti-bacterial, anti-fungal, and anti-viral properties via complex signaling [6]. GM-CSF has been used as an adjuvant for diverse anti-cancer therapies including immune therapy and anti-cancer vaccines [7-9]. Other uses of GM-CSF include therapy of post-transplant graft failure [10], reversal of immune paralysis (i.e., persistence of a marked compensatory anti-inflammatory innate immune response following an insult such as sepsis or trauma) [11-15], and treatment of lung diseases such as autoimmune pulmonary alveolar proteinosis, acute respiratory distress syndrome (ARDS), and pneumonia $[11,16]$.

In an uncontrolled clinical trial, Herold and colleagues [11] gave aerosolized GM-CSF (sargramostim), $125 \mu \mathrm{g}$ for 2 doses $48 \mathrm{~h}$ apart to 6 subjects with moderate-to-severe community-acquired pneumonia or ventilator-associated ARDS. They reported improved oxygenation in subjects receiving GM-CSF compared with controls with a mean increase of about $40 \%$ in lung compliance [11]. GM-CSF promoted an M1 phenotype of alveolar macrophages and increased activation of alveolar mononuclear phagocytes without increasing neutrophils in the alveolar compartment. Similarly, safety of aerosolized sargramostim was reported in autoimmune pulmonary alveolar proteinosis [16]. A phase-2 Belgian, multi-center SARPAC study used aerosolized sargramostim in persons with COVID-19-related ARDS (EudraCT 2020-00125422, NCT04326920) [17]. Preliminary data are most encouraging (unpublished observations).

Hematologists and oncologists are more familiar with G-CSF than GM-CSF, and as shown in Figure 1, G-CSF accounts for $>95 \%$ of the use of molecularly cloned myeloid hematopoietic growth factors. Consequently, many physicians may be more likely to use G-CSF than GMCSF in persons with COVID-19-related ARDS. This may be a mistake based on the data we cite regarding G-CSFinduced influx of granulocytes in the lung, an effect not seen with GM-CSF. However, there are no comparative clinical data in this setting (see below).

Whether aerosolized molecularly cloned hematopoietic growth factors are safe and effective in COVID19-related ARDS and in other SARS-CoV-2-infected persons is controversial and unknown. Several reports suggest not giving molecularly cloned hematopoietic growth factors to persons undergoing conventional chemotherapy and hematopoietic cell transplantation during the SARS-CoV-2 pandemic because of concerns of increasing lung inflammation or the hypothetical risk of increasing inflammatory cytokines such as interleukin-6 (IL-6) associated with an adverse outcome $[18,19]$. However, these recommendations are not evidenced-based and there are no published data reporting such events in humans.

Several studies report systemic G-CSF can exacerbate lung injury in the setting of pulmonary infection. For example, Jing and colleagues [20] reported G-CSF increases lung injury in a mouse model of acute renal injury. Wang et al. $[21,22]$ reported blocking the G-CSF receptor in mouse models of infection and asthma reduced neutrophil infiltration and neutrophil-mediated inflammation. Tsantikos and associates [23] reported G-CSF was important in the pathogenesis of chronic obstructive pulmonary disease in some persons. Arimura and co-workers [24] described severe acute lung injury in a healthy hematopoietic cell transplant donor given G-CSF. Boujoukos and colleagues [25] reported that during the initial inflammatory response to endotoxin in humans, the alveolar space is relatively insulated from cytokine-induced effects of endotoxin including tumor necrosis factor, IL-6, and IL- 8 but not G-CSF. Takatsuka et al. [26] reported five people developed ARDS whilst receiving G-CSF with chemotherapy or a hematopoietic cell transplant. These data suggest that giving G-CSF can worsen lung function by causing neutrophil infiltration. This effect is especially so in settings of inflammation such as infection and cytokine release syndrome. Because cytokine release syndrome is a feature of COVID-19-related ARDS, caution is needed.

These same adverse effects on lung function are not reported in mouse models or humans receiving sargramostim in similar settings. The favorable preliminary 
data from the SARPAC study using aerosolized sargramostim are encouraging [17]. Several studies suggest G-CSF is likely to exacerbate lung injury in the setting of infection. Consequently, persons receiving intensive chemotherapy during the SARS-CoV-2 pandemic, especially those with COVID-19, may not be receiving GCSF. Giving GM-CSF may be associated with less lung injury risk. Three clinical trials of GM-CSF in persons with COVID-19-related ARDS are in progress (SARPAC [EudraCT 2020-001254-22; NCT04326920], iLeukPulm [NCT04411680], and NCT04400929).

In summary, although G-CSF and GM-CSF are molecularly cloned myeloid growth factors, their biology and clinical effects differ. GM-CSF has a much wider activity spectrum in animals and humans. In persons with lung infection and/or ARDS, GM-CSF may be a safer drug than G-CSF. Whether this is so can only be definitively answered in a randomized comparison trial. Unfortunately, this is unlikely to be done and we may have to rely on indirect evidence of safety and efficacy.

\section{Acknowledgement}

We thank Luis Leal, DPM and Carolyn Ragsdale, PharmD, who kindly reviewed the typescript. R.P.G. acknowledges support from the National Institute of Health Research (NIHR) Biomedical Research Centre funding scheme.

\section{Conflict of Interest Statement}

H.M.L. is a consultant to Partner Therapeutics. R.P.G. received funds from Partner Therapeutics for consulting within the past 2 years but none in relation to this publication.

\section{Funding Sources}

Partner Therapeutics financially supported this publication.

\section{Author Contributions}

H.M.L. and R.P.G. equally contributed to the entirety of this publication.

\section{References}

1 Smith TJ, Bohlke K, Lyman GH, Carson KR, Crawford J, Cross SJ, et al.; American Society of Clinical Oncology. Recommendations for the use of WBC growth factors: American Society of Clinical Oncology clinical practice guideline update. J Clin Oncol. 2015 Oct; 33(28):3199-212.

2 Gale RP, Armitage JO. Use of molecularlycloned haematopoietic growth factors in persons exposed to acute high-dose, high-dose rate whole-body ionizing radiations. Blood Rev. 2020 Apr 2:100690.

3 Dorr RT. Clinical properties of yeast-derived versus Escherichia coli-derived granulocytemacrophage colony-stimulating factor. Clin Ther. 1993 Jan-Feb;15(1):19-29.

4 Bhattacharya P, Thiruppathi M, Elshabrawy HA, Alharshawi K, Kumar P, Prabhakar BS. GM-CSF: an immune modulatory cytokine that can suppress autoimmunity. Cytokine. 2015 Oct; $75(2): 261-71$

5 Dos Santos Cruz GJ, Loraine H, Zhang JG, Clark KL, Davis RJ. G-CSF and GM-CSF are differentially released from primary human lung cells in response to pro-inflammatory cytokines (abstract). Pharmagene Laboratories Ltd, 2 Orchard Road, Royston, SG8 5HD. UK. Available at: https://www.veritastk.co.jp/ products/images/G-CSF\%20and\%20GMCSF $\% 20$ are $\% 20$ Differentially\%20Released\%20from.pdf

6 Damiani G, McCormick TS, Leal LO, Ghannoum MA. Recombinant human granulocyte macrophage-colony stimulating factor expressed in yeast (sargramostim): A potential ally to combat serious infections. Clin Immunol. 2020 Jan;210:108292.

7 Nahas MR, Rosenblatt J, Lazarus HM, Avigan D. Anti-cancer vaccine therapy for hematologic malignancies: an evolving era. Blood Rev. 2018 Jul;32(4):312-25.

8 McMasters M, Blair BM, Lazarus HM, Alonso CD. Casting a wider protective net: anti-infective vaccine strategies for patients with hematologic malignancy and blood and marrow transplantation. Blood Rev. [In Press 2020].

9 Kushner BH, Cheung IY, Modak S, Basu EM, Roberts SS, Cheung NK. Humanized 3F8 anti-GD2 monoclonal antibody dosing with granulocyte-macrophage colony-stimulating factor in patients with resistant neuroblastoma: A phase 1 clinical trial. JAMA Oncol. 2018 Dec;4(12):1729-35.

10 Weisdorf DJ, Verfaillie CM, Davies SM, Filipovich AH, Wagner JE Jr, Miller JS, et al. Hematopoietic growth factors for graft failure after bone marrow transplantation: a randomized trial of granulocyte-macrophage colony-stimulating factor (GM-CSF) versus sequential GM-CSF plus granulocyte-CSF. Blood. 1995 Jun;85(12):3452-6.

11 Herold S, Hoegner K, Vadász I, Gessler T, Wilhelm J, Mayer K, et al. Inhaled granulocyte/macrophage colony-stimulating factor as treatment of pneumonia-associated acute respiratory distress syndrome. Am J Respir Crit Care Med. 2014 Mar;189(5):609-11.

12 Hall MW. Immune modulation in pediatric sepsis. J Pediatr Intensive Care. 2019 Mar; $8(1): 42-50$.
13 Mathias B, Szpila BE, Moore FA, Efron PA Moldawer LL. A review of GM-CSF therapy in sepsis. Medicine (Baltimore). 2015 Dec; 94(50):e2044

14 Meisel C, Schefold JC, Pschowski R, Baumann T, Hetzger K, Gregor J, et al. Granulocyte-macrophage colony-stimulating factor to reverse sepsis-associated immunosuppression: a double-blind, randomized, placebo-controlled multicenter trial. Am J Respir Crit Care Med. 2009 Oct;180(7): 640-8.

15 Paine R 3rd, Standiford TJ, Dechert RE, Moss M, Martin GS, Rosenberg AL, et al. A randomized trial of recombinant human granulocyte-macrophage colony stimulating factor for patients with acute lung injury. Crit Care Med. 2012 Jan;40(1):90-7.

16 Tazawa R, Ueda T, Abe M, Tatsumi K, Eda R, Kondoh S, et al. Inhaled GM-CSF for pulmonary alveolar proteinosis. N Engl J Med. 2019 Sep;381(10):923-32.

17 Bosteels C, Maes B, Van Damme K, De Leeuw E, Declercq J, Delporte A, et al. Sargramostim to treat patients with acute hypoxic respiratory failure due to COVID-19 (SARPAC): A structured summary of a study protocol for a randomised controlled trial. Trials. 2020 Jun; 21(1):491.

18 Malard F, Genthon A, Brissot E, van de Wyngaert Z, Marjanovic Z, Ikhlef S, et al. COVID-19 outcomes in patients with hematologic disease. Bone Marrow Transplant. 2020 May; Epub ahead of print. https://doi.org/10.1038/ s41409-020-0931-4. 
19 Ljungman P, Mikulska M, de la Camara R, Basak GW, Chabannon C, Corbacioglu S, et al. The challenge of COVID-19 and hematopoietic cell transplantation; EBMT recommendations for management of hematopoietic cell transplant recipients, their donors, and patients undergoing CAR T-cell therapy [Epub ahead of print]. Bone Marrow Transplant. 2020 May 13:1-6.

20 Jing W, Qin F, Guo X, Sun Y, Yan C, Qiu C, et al. G-CSF mediates lung injury in mice with adenine-induced acute kidney injury. Int Immunopharmacol. 2018 Oct;63:1-8.
21 Wang H, FitzPatrick M, Wilson NJ, Anthony D, Reading PC, Satzke C, et al. CSF3R/CD114 mediates infection-dependent transition to severe asthma. J Allergy Clin Immunol. 2019 Feb;143(2):785-788.e6.

22 Wang H, Aloe C, Wilson N, Bozinovski S. GCSFR antagonism reduces neutrophilic inflammation during pneumococcal and influenza respiratory infections without compromising clearance. Sci Rep. 2019 Nov;9(1): 17732.

23 Tsantikos E, Lau M, Castelino CM, Maxwell MJ, Passey SL, Hansen MJ, et al. GranulocyteCSF links destructive inflammation and comorbidities in obstructive lung disease. J Clin Invest. 2018 Jun;128(6):2406-18.
24 Arimura K, Inoue H, Kukita T, Matsushita K, Akimot M, Kawamata N, et al. Acute lung Injury in a healthy donor during mobilization of peripheral blood stem cells using granulocyte-colony stimulating factor alone. Haematologica. 2005 Mar;90(3):ECR10.

25 Boujoukos AJ, Martich GD, Supinski E, Suffredini AF. Compartmentalization of the acute cytokine response in humans after intravenous endotoxin administration. J Appl Physiol (1985). 1993 Jun;74(6):3027-33.

26 Takatsuka H, Takemoto Y, Mori A, Okamoto T, Kanamaru A, Kakishita E. Common features in the onset of ARDS after administration of granulocyte colony-stimulating factor. Chest. 2002 May;121(5):1716-20. 